\title{
SINTOMAS DEPRESSIVOS, AUTORREGULAÇÃO EMOCIONAL E SUPORTE FAMILIAR: UM ESTUDO COM CRIANÇAS E ADOLESCENTES
}

\author{
Lisandra Borges \\ Universidade de São Francisco
}

\author{
Janaína Thais Barbosa Pacheco \\ Universidade Federal de Ciências da Saúde de Porto Alegre
}

\begin{abstract}
Resumo
A depressão infantil acarreta prejuízos em diferentes contextos da vida, tanto das crianças quanto de seus familiares. O objetivo do estudo foi verificar a prevalência de sintomas depressivos para uma amostra de escolares e buscar a relação entre descritores de depressão, autorregulação emocional e percepção do suporte familiar. Como objetivos secundários, buscou-se diferenças entre sexo e idade. Participaram 546 crianças e adolescentes, sendo a maioria $(n=276 ; 50,5 \%)$ meninos, com idades entre 8 a 18 anos $(M=12,65 ; D P=1,84)$. Os instrumentos utilizados foram a Escala Baptista de Depressão Infantil, Bateria de Indicadores de Depressão Infantojuvenil, Escala de Autorregulação Emocional e Inventário de Percepção de Suporte Familiar. Os resultados indicaram prevalência de sintomatologia leve $(4,8 \%)$, moderada $(20,3 \%)$ e severa (15\%). A depressão mostrou-se associada a dificuldades de autorregulação emocional e percepção do suporte familiar. Conclui-se que o entendimento acerca da expressão da sintomatologia depressiva pode contribuir para diagnóstico e intervenção precoces.
\end{abstract}

Palavras-chave: depressão infantil; avaliação psicológica; saúde mental infantil.

\section{DEPRESSIVE SYMPTOMS, EMOTIONAL SELF-REGULATION AND FAMILY SUPPORT: A STUDY WITH CHILDREN AND ADOLESCENTS}

\begin{abstract}
Child depression leads to losses in different contexts of life, for both children and their families. The aim of the study was to verify the prevalence of depressive symptoms for a sample of schoolchildren and to search for the relationship between descriptors of depression, emotional self-regulation and perception of family support. As secondary objectives, we sought differences between sex and age. There were 546 children and adolescents, the majority $(n=276,50.5 \%)$ boys, aged 8 to 18 years $(M=12.65, S D$ $=1.84$ ). The instruments used were the Baptist Scale for Child Depression, the Battery for Children's Depression Indicators, the Emotional Self-Regulation Scale, and the Family Support Perception Inventory. The results indicated a prevalence of mild $(4.8 \%)$, moderate $(20.3 \%)$ and severe $(15 \%)$ symptomatology. Depression was associated with difficulties of emotional self-regulation and perception of family support. It is concluded that the understanding of the expression of depressive symptomatology can contribute to early diagnosis and intervention.

Keywords: child depression; psychological assessment; child mental health.
\end{abstract}




\title{
SÍNTOMAS DEPRESIVOS, AUTORREGULACIÓN EMOCIONAL Y SOPORTE FAMILIAR: UN ESTUDIO CON NIÑOS Y ADOLESCENTES
}

\begin{abstract}
Resumen
La depresión infantil acarrea perjuicios en diferentes contextos de la vida, tanto de los niños y de sus familiares. El objetivo del estudio fue verificar la prevalencia de síntomas depresivos para una muestra de escolares y buscar la relación entre descriptores de depresión, autorregulación emocional y percepción del soporte familiar. Como objetivos secundarios, se buscaron diferencias entre sexo y edad. En el caso de los niños y adolescentes, la mayoría $(n=276,50,5 \%)$, con edades entre 8 a 18 años $(M=12,65, D P=1,84)$. Los instrumentos utilizados fueron la Escala Baptista de Depresión Infantil, Batería de Indicadores de Depresión Infantojuvenil, Escala de Autorregulación Emocional e Inventario de Percepción de Soporte Familiar. Los resultados indicaron prevalencia de sintomatología leve $(4,8 \%)$, moderada $(20,3 \%)$ y severa $(15 \%)$. La depresión se asoció a dificultades de autorregulación emocional y percepción del soporte familiar. Se concluye que el entendimiento acerca de la expresión de la sintomatología depresiva puede contribuir para el diagnóstico e intervención temprana.
\end{abstract}

Palabras clave: depresión infantil; evaluación psicológica; salud mental infantil.

\section{INTRODUÇÃO}

A depressão tem sido considerada uma das doenças mais incapacitantes do mundo, acometendo pessoas de diferentes classes sociais, etnias e também idades. Os prejuízos decorrentes do transtorno interferem em todos os contextos, seja na escola, trabalho e relacionamentos. As altas taxas de prevalência da depressão têm chamado atenção dos profissionais da área da saúde e também da comunidade cientifica em geral, pois vem sendo considerada a segunda doença mais proeminente entre a população. Trata-se de um transtorno mental comum, sendo que a estimativa mundial é de que mais de 300 milhões de pessoas de sofrem de depressão (World Health Organization, 2017).

De acordo com o Manual Diagnóstico e Estatístico de Transtornos Mentais (DSM-5; American Psychiatric Association [APA], 2014), o transtorno depressivo é caracterizado por tristeza persistente, anedonia (perda de interesse ou prazer em atividades anteriormente consideradas prazerosas), alteração no apetite e no padrão de sono (aumento ou diminuição), dificuldade de concentração e tomada de decisão, sentimento de culpa e inutilidade, desesperança, pensamentos negativos e ideias suicidas, por no mínimo, um período de duas semanas de duração. Importante destacar que o DSM-5 não apresenta critérios diagnósticos específicos para avaliação de crianças e adolescentes.

Diferentes estudos brasileiros têm buscado identificar a prevalência de depressão no público infantojuvenil. Argimon, Terroso, Barbosa e Lopes (2013) avaliaram 88 adolescentes de 12 a 17 anos no Sul e encontraram ocorrência de $23 \%$ sintomas moderados e graves. Couto, Reis e Oliveira (2016) contaram com amostra de 220 estudantes de 11 a 17 anos, destes, 7,72\% apresentaram sintomas de depressão. Thiengo, Cavalcante e Lovisi (2014) realizaram uma revisão na literatura acerca da prevalência, identificando variação de 0,6 a 30\% 
de ocorrência de transtorno depressivo. Mais da metade dos estudos incluídos na revisão indicaram uma prevalência de $5,9 \%$ a $12,5 \%$ de depressão. As diferenças encontradas nos estudos podem ser decorrentes de fatores, como instrumentos de avaliação, faixas etárias investigadas e procedimentos de coleta de dados.

Além disso, as medidas nem sempre são uniformes, pois há na literatura, uma discussão acerca dos sintomas depressivos mais comuns para esse público. É sabido que os fatores relacionados à depressão na infância e na adolescência envolvem aspectos genéticos, individuais e ambientais. Entre esses fatores destacam-se sexo, histórico de transtorno mental familiar, presença de violência comunitária e familiar, configuração e a organização familiar (Thiengo et al., 2014).

Alguns autores (Baptista, Borges, \& Serpa, 2017) têm apontado sintomas característicos para sexo e faixa etária nesta população. Para Baptista et al. (2017), os meninos com idade entre 8 e 12 anos apresentam dificuldade de resolução de problemas, agressividade, baixa concentração, ideação suicida, baixa sociabilidade e solidão. Já as meninas com a mesma faixa etária apresentam maior anedonia, alteração no apetite, baixa autoestima, desesperança, rebaixamento de humor, sociabilidade reduzida, choro e vontade de chorar. Alguns autores (Avanci, Assis, \& Oliveira, 2008; Baptista, 2017; Borges, Baptista, \& Serpa, 2017) também têm encontrado diferenças em relação ao sexo, sendo que, a partir da adolescência, as meninas apresentam maiores escores quando comparadas aos meninos. As diferenças entre os sexos podem estar relacionadas à socialização, aos hormônios e a eventos estressantes associados à adolescência (Luby, Gaffrey, Tillman, Abril, \& Belden, 2014). De acordo com Baptista (2017) e Girgus e Yang (2015), esses estressores decorrem de práticas sociais e educativas de poder e controle, abuso físico, gestão emocional e estratégias de enfrentamento, pensamentos negativos, orientação sexual e imagem corporal negativa.

Compreende-se que a sintomatologia depressiva na população infantojuvenil nem sempre é de fácil observação, pois inclui aspectos internalizantes, dificultando sua observação e possível diagnóstico, o que justifica cada vez mais novos estudos que busquem a compreensão do fenômeno. Para Borges et al. (2017), a depressão infantil é considerada um construto multidimensional. Nesse sentido, têm apontado a necessidade de avaliar alguns indicadores que estão relacionados à depressão, o que pode contribuir para um diagnóstico precoce e possível adequado. Os indicadores (sintomas principais da depressão, solidão, desamparo, autoestima, autoconceito, desesperança e autoeficácia) serviram de base para construção da Bateria de Avaliação de Indicadores de Depressão Infantojuvenil -BAID-IJ (Borges, 2015).

Alguns estudiosos, preocupados em compreender a complexidade da depressão na infância, têm direcionado sua atenção para a forma como as 
crianças com sintomatologia depressiva regulam suas emoções. Há pesquisas sugerindo que crianças com depressão não empregam de maneira eficiente estratégias de regulação afetiva (Burwell \& Shirk, 2007; Joorman \& Gotlib, 2010). Para Peterson e Seligman (2004), a autorregulação diz respeito à forma como uma pessoa controla suas próprias respostas para alcançar suas metas e objetivos. Tais respostas incluem pensamentos, emoções, impulsos, e outros comportamentos. Dessa forma, segundo os autores, as pessoas podem direcionar seus processos de pensamento, mudando respostas emocionais que as impediriam de realizar seus desejos e/ou atingir suas metas. Elas podem tentar fazer melhor do que normalmente fariam, como por exemplo, persistir em uma tarefa difícil.

Além disso, para alguns autores (Howell, Digdon, \& Buro, 2010; Weiss, Gratz, \& Lavender, 2015), pessoas com dificuldade de se autorregular emocionalmente podem apresentar dificuldades como ansiedade, depressão, problemas com o sono e comportamentos autodestrutivos. Assim sendo, a identificação do nível de autorregulação emocional de uma pessoa pode ser um recurso importante para se compreender a origem dos problemas emocionais ou psicológicos e assim, elaborar uma intervenção mais adequada (Nelis, Quoidbach, Hansenne, \& Mikolajczak, 2011).

Como afirmam Joorman e Gotlib (2010), surpreendentemente, poucos estudos examinaram o uso de estratégias de regulação emocional na depressão. No Brasil, Coutinho, Ribeiro, Ferreirinha e Dias (2010) avaliaram uma amostra composta por 343 adolescentes e adultos. Os resultados mostraram correlação positiva $(\rho=0,58 ; p<, 001)$ e significativa, indicando que quanto maior a dificuldade em controlar as emoções, maior a apresentação de sintomas depressivos.

Cruvinel e Boruchovitch (2011) investigaram a regulação emocional de 54 crianças com e sem diagnóstico de depressão. Os resultados apontaram que o grupo com diagnóstico apresentou maior dificuldade ao expressar emoções se comparado ao grupo sem diagnóstico, além de fazerem mal-uso das emoções negativas. Nesse mesmo sentido, Porro, Andrés e Rodrígues-Espínola (2012) avaliaram uma amostra de 38 pacientes oncológicos e 44 pessoas sem diagnóstico. Os resultados mostraram que os grupos se diferenciaram significativamente na frequência no uso da expressão emocional, sendo que o os pacientes diagnosticados expressaram menos suas emoções negativas.

Dentre outros fatores relacionados à depressão na infância e adolescência, destacam-se a presença de violência comunitária e familiar, configuração e a organização familiar (Thiengo et al., 2014). A esses aspectos, é possível destacar a importância da família sob diferentes perspectivas como um fator de risco ou de proteção para o adolescente. Há consenso na literatura sobre a importância da adequada percepção do suporte familiar, para o desenvolvimento biopsicossocial da criança e do adolescente, sendo considerado também como 
um fator protetivo aos aspectos ligados aos transtornos afetivos em crianças, adolescentes e adultos (Dessen, 2010; Gonçalves, Baptista, \& Farcas, 2016).

Para Baptista, Baptista, e Dias (2001), a percepção do suporte familiar pode ter relação direta com o modo como a pessoa avalia a si mesma e as informações do meio em que está inserida. O suporte fornecido e recebido pela família, por meio dos padrões de relacionamento e de comunicação entre seus membros, pode ser considerado um amortecedor dos efeitos de estressores, tendendo a favorecer a competência social, estratégias de enfrentamento das dificuldades da vida, senso de estabilidade, afeto positivo, bem-estar psicológico e o autoconceito positivo (Rigotto, 2006; Santos, 2006). Estudos anteriores indicaram relações entre suporte familiar e sintomatologia depressiva, estratégias de enfrentamento e percepção de carinho e de relações de colaboração e compreensão (Baptista, Carneiro \& Sisto, 2010). O estudo de Baptista, Souza, e Alves (2008) avaliou 157 estudantes por meio de uma escala de depressão e o IPSF. Os autores encontraram correlações negativas entre os construtos ( $-0,36$ afetivo-consistente; $-0,37$ adaptação familiar; $-0,32$ autonomia familiar e $-0,42$ suporte total.

Diante do exposto e da necessidade da compreensão da manifestação dos sintomas depressivos em crianças e adolescentes, o presente estudo tem como objetivo geral verificar a prevalência de sintomas depressivos para amostra pesquisada. Ademais, busca investigar a associação entre indicadores de depressão, autorregulação emocional e percepção de suporte familiar. Além disso, explorou-se possíveis diferenças em relação às variáveis sexo e idade.

\section{MÉTODO}

\section{Participantes}

A amostra foi composta por 546 crianças e adolescentes ( $n=276 ; 50,5 \%$ do sexo masculino) de escolas públicas de cidades do interior de São Paulo e de Porto Alegre. A idade variou entre 8 e 18 anos, com média de 12,65 anos ( $D P=$ $1,84)$.

\section{Instrumentos}

Escala Baptista de Depressão - Versão Infantojuvenil (EBADEP-IJ, Baptista, 2017).

Trata-se de um instrumento constituído por 27 itens, que tem como objetivo avaliar a sintomatologia depressiva em crianças e adolescentes. É composto por uma escala do tipo Likert, de três pontos, variando de zero a dois, com pontuação mínima de zero e máxima de 100 pontos. O instrumento é baseado nos descritores do DSM-5 (APA, 2014), CID-10 (Organização Mundial de Saúde [OMS], 2000), Terapia Cognitiva (Beck, Rush, Shaw, \& Emery, 1997), 
Princípios do Comportamento (Ferster, Culbertson, \& Boren, 1977), bem como nos estudos de Weinberg, Rutman, Sullivan, Pencik e Dietz (1973), e apresenta 24 descritores, que são: humor deprimido, perda ou diminuição de prazer, choro, desesperança, desamparo, indecisão, sentimento de incapacidade e inadequação, carência ou dependência, negativismo, esquiva de situações sociais, queda de produtividade, inutilidade, autocrítica exacerbada, culpa, diminuição da concentração, pensamento de morte, autoestima rebaixada, falta de perspectiva sobre o presente, alteração de apetite, alteração de peso, insônia ou hipersonia, lentidão ou agitação psicomotora, fadiga e irritação. Exemplos de itens: Meus dias têm sido bons; Tenho dormido bem; Sinto-me sem energia.

Bateria de Avaliação de Indicadores de Depressão Infantojuvenil (BAID-IJ; Borges, \& Baptista, 2015).

A BAID-IJ contém 109 itens que se propõem a avaliar depressão (18 itens), solidão (13 itens), desamparo (17 itens), autoestima (18 itens), autoconceito (16), desesperança (14 itens) e autoeficácia (13 itens) distribuídos em cinco escalas em formato do tipo Likert de três pontos "Não/Nunca; "Às vezes" e "Sim/Sempre". É pedido ao respondente que leve em consideração seus pensamentos, sentimentos e comportamento nos últimos 15 dias. As frases das três primeiras escalas, depressão, solidão e desamparo foram construídas de forma negativa, ou seja, os itens priorizam sentimentos, pensamentos e comportamentos negativos, como exemplo "sinto-me triste", "sinto que as pessoas me evitam" e "mesmo fazendo coisas diferentes, meus dias serão chatos". A s outras duas escalas, autoestima e autoconceito foram construídas de forma positiva, como exemplo "sou útil" e "meus professores acham que sou inteligente". A criança ou adolescente assinala cada frase com um X, a opção que melhor explica.

Escala de Autorregulação Emocional (EARE; Noronha \& Baptista, 2016).

Trata-se de uma escala de avaliação do controle expressivo das emoções, envolvendo $o$ monitoramento e avaliação das experiências emocionais destacando a importância de estar atento as emoções. A escala é embasada nos pressupostos teóricos de Gratz e Roemer (2004), Nelis et al. (2011), Weiss et al. (2015). O objetivo do instrumento é avaliar a autorregulação emocional diante de algum evento que gera tristeza. Ao responder, a pessoa deve considerar 0 quanto seus pensamentos, sentimentos e comportamentos melhor o descrevem quando está triste. A versão (preliminar) do instrumento utilizada é composta por 84 itens, dispostos em uma escala do tipo Likert de 5 pontos $(0=$ nunca e $4=$ sempre). Quanto maior a pontuação, maior o nível de autorregulação emocional do respondente. Os fatores da escala são: estratégias de enfrentamento adequadas/avaliação da experiência; paralisação; pessimismo; e externalização da agressividade/agressividade externalizada. Exemplo de item: "Acho que o que 
sinto é importante para meu crescimento" "Não sei o que fazer" "Acredito que tudo vai piorar". No presente estudo foi utilizado o escore total do instrumento.

Inventário de Percepção do Suporte Familiar (IPSF; Baptista, 2009).

O IPSF tem como objetivo avaliar como o indivíduo percebe o suporte que recebe da sua família. O inventário é composto por 42 afirmações relacionadas a situações familiares, em que o participante deve marcar a frequência com que cada uma delas acontece em sua família. As respostas são do tipo Likert de três pontos, possuindo três dimensões: afetivo consistente, (relações afetivas positivas, carinho, proximidade, clareza em papéis e regras dos integrantes da família e habilidade na resolução de problemas); adaptação familiar (sentimentos negativos sobre a família, tais como raiva, isolamento, exclusão, vergonha, incompreensão e interesse; e autonomia familiar (relações de confiança, liberdade e privacidade).

\section{Procedimentos}

Inicialmente solicitou-se carta de autorização das escolas para coleta de dados e o projeto foi encaminhado ao Comitê de Ética em Pesquisa de uma instituição de ensino superior (CAAE: 60623516.7.0000.5514). A coleta de dados foi agendada de acordo com a disponibilidade de cada escola. Os pais ou responsáveis assinaram o Termo de Consentimento Livre e Esclarecido (TCLE) autorizando a participação das crianças e adolescentes. No momento da coleta, os objetivos da pesquisa foram explicados e os participantes assinaram o Termo de Assentimento Livre e Esclarecido (TALE). A coleta foi realizada de forma coletiva, em sala de aula, conforme disponibilidade das escolas, com tempo aproximado de 60 minutos.

\section{Plano de análise dos dados}

Os dados foram submetidos às estatísticas descritivas e inferenciais por meio do programa Statistical Package for the Social Science (SPSS). Averiguouse que os dados obtidos nesta amostra não possuíam uma distribuição normal (testada pelos métodos de Kolmogorov-Smirnov e Shapiro-Wilk), optando-se pelo uso da estatística não paramétrica. Em ambos, o valor de $p$ foi $<0,05$ caracterizando a distribuição não normal para a maioria das variáveis. Apenas as variáveis autoeficácia e autorregulação apresentaram distribuição normal $(p>0,05)$ na amostra. Para responder aos objetivos do estudo foram utilizadas as estatísticas não paramétricas: teste de correlação de Spearman para obter o $\rho$ (rho) para investigar a relação entre as variáveis estudadas e teste de KruskalWallis e o teste U de Mann-Whitney para comparação entre grupos. 


\section{RESULTADOS E DISCUSSÃO}

Para maior clareza dos resultados encontrados, optou-se incialmente por descrever as pontuações (mínima, máxima, média e desvio padrão) de cada variável pesquisada. A Tabela 1 apresenta as estatísticas descritivas dos instrumentos BAID-IJ, EARE e IPSF.

Tabela 1

Estatística descritiva da BAID, IPSF e EARE.

\begin{tabular}{ccccc}
\hline Variáveis (amplitude) & Mínimo & Máximo & Média & Desvio-Padrão \\
\hline Depressão (0-36) & 0 & 35 & 9,70 & 7,27 \\
Solidão (0-26) & 0 & 21 & 4,42 & 4,76 \\
Desamparo (0-34) & 0 & 27 & 9,35 & 6,49 \\
Autoestima (0-36) & 10 & 36 & 26,54 & 6,38 \\
Autoconceito (0-32) & 5 & 32 & 24,79 & 5,60 \\
Desesperança (0-28) & 0 & 20 & 8,45 & 4,42 \\
Autoeficácia (0-30) & 9 & 30 & 21,08 & 4,73 \\
Autorregulação & 0 & 97 & 44,62 & 16,48 \\
Afetivo-Consistente & 1 & 42 & 25,92 & 8,09 \\
Adaptação & 3 & 40 & 20,52 & 5,25 \\
Autonomia & 0 & 16 & 8,66 & 3,35 \\
IPSF Total & 8 & 83 & 55,22 & 12,95 \\
\hline
\end{tabular}

De acordo com o primeiro objetivo do estudo, apontar a prevalência de sintomas depressivos, optou-se por utilizar dados referentes a EBADEP-IJ, por se tratar de um instrumento que apresenta normas para população. Dessa forma, considerou-se, de acordo com Baptista (2017), o intervalo entre 21 e 30 pontos brutos, sendo interpretado como sintomatologia leve; entre 31 e 45 pontos brutos, sendo interpretado como sintomatologia moderada e por fim, o intervalo entre 46 e 54 pontos brutos, sendo interpretado como sintomatologia grave ou severa. Os resultados possibilitam identificar que para a amostra pesquisada, $22 \%(n=121)$ dos participantes não apresentou sintomas depressivos, 4,8\% $(n=26)$ apresentou sintomas leves, e 20,3\% $(n=111)$ apresentou sintomas moderados e $15 \%(n=86)$ dos participantes apresentou sintomas severos.

Em conformidade com os achados na literatura, por um lado, pode-se identificar certa discrepância em relação aos dados. Por outro lado, a maioria aponta alta prevalência de sintomas depressivos para as amostras pesquisadas. Argimon et al. (2013) encontraram $23 \%$ de sintomas moderados e graves, enquanto Couto et al. (2016), identificaram 7,72\%. A variação da prevalência encontrada no estudo de revisão de Thiengo et al. (2014) foi de 0,6 a 30\%. A prevalência encontrada no presente estudo foi de $40,10 \%$, resultado que pode 
ser considerado alto em relação aos demais. Nesse sentido, concorda-se com o pensamento de Borges e Baptista (2015) e Thiengo et al. (2014), de que não há como comparar tais dados de prevalência, primeiro porque são regionalizados, os instrumentos de medida utilizados apresentam diferentes perspectivas, bem como as faixas etárias e os procedimentos de coleta de dados apresentam variação.

A Tabela 2 apresenta a análise da correlação de Spearman. Todas as variáveis descritas na Tabela 1 foram inseridas na análise de correlação, no entanto, serão apresentadas apenas as que foram estatisticamente significativas e acima de 0,30 $(\rho>0,30)$. A idade dos participantes da amostra também foi incluída na análise, no entanto, não foram encontrados resultados estatisticamente significativos.

Tabela 2

Correlação ( $\rho$ de Spearman) entre as variáveis da BAID, IPSF e EARE.

\begin{tabular}{cccccccccccc}
\hline & 1 & 2 & 3 & 4 & 5 & 6 & 7 & 8 & 9 & 10 & 11 \\
\hline 2 & .68 & & & & & & & & & & \\
3 & .49 & .58 & & & & & & & & & \\
4 & & -.34 & -.40 & & & & & & & & \\
5 & -36 & -.40 & -.41 & .71 & & & & & & & \\
6 & .53 & .47 & .60 & -.45 & & & & & & & \\
7 & -.43 & .47 & -.54 & .52 & .67 & -.31 & & & & & \\
8 & .71 & & .48 & & & .34 & & & & & \\
9 & -.23 & & -.52 & & & & & & & & \\
10 & -.60 & -.46 & -.67 & & & -.42 & .31 & -.33 & .37 & & \\
11 & & & .30 & .38 & & & & & .43 & & \\
12 & .40 & .30 & .64 & .35 & & & .31 & & .91 & .61 & .60 \\
\hline
\end{tabular}

Nota. 1. Depressão; 2. Solidão; 3. Desamparo; 4. Autoestima; 5. Autoconceito; 6. Desesperança; 7. Autoeficácia; 8. Autorregulação; 9. Afetivo-consistente; 10. Adaptação; 11. Autonomia; 12. IPSF; As correlações em negrito apresentaram $p$ $<0,05$ e as demais $p<0,01$.

Diante dos resultados foi possível identificar algumas correlações importantes entre indicadores de depressão e autorregulação emocional. A correlação $\rho=0,71$, considerada de magnitude forte (Dancey \& Reid, 2013) foi significativa entre sintomas principais da depressão e da autorregulação emocional, indicando que quanto maior a expressão de sintomas depressivos, maiores as dificuldades em lidar com as questões emocionais. Foram encontrados ainda $\rho=0,48$ (desamparo) e $\rho=0,34$ (desesperança). Para Burwell e Shirk, (2007) e Joorman e Gotlib (2010), as pesquisas sugerem que crianças 
com depressão não empregam de maneira eficiente estratégias de regulação afetiva.

Apesar de escassos os estudos que buscam tal relação, os resultados vão ao encontro dos achados de Cruvinel e Boruchovitch (2011), que chamaram atenção para o fato de que crianças que experimentam sentimentos como tristeza e raiva com maior frequência têm maior dificuldade em perceber suas emoções. A literatura aponta que pessoas com estratégias de autorregulação emocional compreendem e aceitam melhor suas emoções, controlam comportamentos impulsivos, vivenciam emoções positivas e negativas sem dificuldades e conseguem modular a intensidade de respostas emocionais (Weiss et al., 2015). Nesse sentido, compreende-se a necessidade de programas que viabilizem o aprendizado de estratégias para que crianças possam lidar suas repostas emocionais, o que certamente auxiliará no desenvolvimento da sua saúde mental

Nas correlações entre a os descritores da BAID-IJ e as dimensões do IPSF, os resultados apontam para uma associação negativa, na medida em que, quanto maior o escore na BAID-IJ, maior a sintomatologia, e quanto maior a pontuação no Inventário de Percepção de Suporte Familiar, melhor o suporte familiar percebido. Não se esperavam correlações altas, uma vez que os construtos envolvidos estão relacionados, porém são diferentes; e como esperado, as correlações foram negativas moderadas. Esse resultado sugere que quanto maior a sintomatologia de depressão, menor o suporte familiar percebido.

As correlações envolvendo as dimensões do IPSF sugerem que quanto maior a percepção de afeto, carinho, interesse, atenção, diálogo, inclusão, compreensão, clareza de papéis e regras familiares, independência e habilidade na resolução de situações-problema, menor a sintomatologia depressiva apresentada pelos participantes. Tais resultados foram ao encontro da literatura. Baptista et al. (2008) encontraram correlações entre sintomas depressivos e percepção do suporte familiar, com correlações negativas e baixas $(-0,36$ afetivo-consistente; $-0,37$ adaptação familiar; $-0,32$ autonomia familiar e $-0,42$ suporte total.

Com o objetivo de explorar os dados e contribuir para a compreensão do fenômeno da depressão na infância e na adolescência, utilizou teste $U$ de MannWhitney e Teste $t$ de Student. Com isso, procedeu-se a verificação de diferença entre os sexos (masculino e feminino); entre grupos por idades (Grupo 1, participantes com idade entre 8 e 12; e Grupo 2, participantes com idade entre 12 e 18 anos); e entre grupos por nível de sintomas de depressão, avaliado pela BAID. Para essa última análise, a amostra foi dividida utilizando-se a mediana encontrada na escala Depressão da BAID-IJ e cujo valor foi oito, constituindo-se dois grupos: Grupo 1, participantes com sintomas de depressão menor do que oito; e Grupo 2, participantes com sintomas de depressão maior do que oito. A 
Tabela 3 apresenta os resultados da análise de comparação entre os grupos com relação aos sintomas de depressão.

Tabela 3

Comparação entre Grupos com diferentes níveis de depressão e as variáveis solidão, desamparo, desesperança, afetivo consistente e adaptação.

\begin{tabular}{|c|c|c|c|c|c|}
\hline Variáveis & $\begin{array}{c}\text { Grupos } \\
\text { Depressão }\end{array}$ & $n$ & Médias & $U$ & $p$ \\
\hline \multirow[t]{3}{*}{ Solidão } & 1 & 113 & 2,20 & 1486* & 0,001 \\
\hline & 2 & 79 & 7,62 & & \\
\hline & Total & 192 & & & \\
\hline \multirow[t]{3}{*}{ Desamparo } & 1 & 24 & 7,62 & $199 *$ & 0,05 \\
\hline & 2 & 27 & 12,33 & & \\
\hline & Total & 51 & & & \\
\hline \multirow[t]{3}{*}{ Desesperança } & 1 & 24 & 9,87 & $156^{*}$ & 0,001 \\
\hline & 2 & 27 & 12,66 & & \\
\hline & Total & 51 & & & \\
\hline \multirow{3}{*}{$\begin{array}{c}\text { Afetivo } \\
\text { Consistente }\end{array}$} & 1 & 113 & 28,04 & $3327,5^{*}$ & 0,001 \\
\hline & 2 & 79 & 24,77 & & \\
\hline & Total & 192 & & & \\
\hline \multirow[t]{3}{*}{ Adaptação } & 1 & 113 & 23,33 & $1855^{*}$ & 0,001 \\
\hline & 2 & 80 & 18,33 & & \\
\hline & Total & 193 & & & \\
\hline \multirow[t]{3}{*}{ IPSF Total } & 1 & 113 & 60,18 & $2534,5^{*}$ & 0,001 \\
\hline & 2 & 79 & 51,18 & & \\
\hline & Total & 192 & & & \\
\hline
\end{tabular}

Como as variáveis autoeficácia e autorregulação apresentaram uma distribuição normal na amostra, procedeu-se a mesma análise, porém utilizando Teste $t$ de Student. Os resultados são apresentados na Tabela 4.

Tabela 4

Comparação entre Grupos com diferentes níveis de depressão e as variáveis autoeficacia e autorregulação

\begin{tabular}{cccccc}
\hline & $\begin{array}{c}\text { Grupos } \\
\text { Depressão }\end{array}$ & $n$ & Média & $t$ & $p$ \\
\hline Autoeficácia & 1 & 49 & 22,81 & 3,76 & 0,001 \\
& 2 & 59 & 19,54 & & \\
Autorregulação & 1 & 108 & & & \\
& 2 & 24 & 35 & 5,39 & 0,001 \\
& Total & 32 & 57,68 & & \\
& Total & 56 & & & \\
\hline
\end{tabular}


Os resultados possibilitam entendimentos em relação ao fenômeno da depressão na infância e na adolescência. Apesar de não terem sido encontradas explorações semelhantes na literatura científica, é possível identificar que o G2 caracterizado pela amostra que apresenta maior sintomas depressivos, pontuaram mais em solidão, desamparo, desesperança, apresentando maiores dificuldades na autorregulação emocional. Em contrapartida, o G1 sem sintomas, apresentou maior autoeficácia e maior percepção do suporte familiar.

A análise de comparação de médias das variáveis investigadas entre os grupos divididos por idades (Grupo 1, participantes com idade entre 8 e 12; e Grupo 2, participantes com idade entre 12 e 18 anos) indicou diferenças significativas em depressão, afetivo-consistente, adaptação familiar e IPSF total (Tabela 5).

Tabela 5

Comparação entre os grupos divididos por idade

\begin{tabular}{cccccc}
\hline & $\begin{array}{c}\text { Grupos por } \\
\text { idade }\end{array}$ & $n$ & Medias & $U$ & $p$ \\
\hline Depressão & 1 & 147 & 8,65 & 6139 & 0,05 \\
& 2 & 103 & 11,20 & & \\
Afetivo & 1 & 250 & & & \\
Consistente & 2 & 202 & 26,9406 & 10217 & 0,05 \\
& Total & 326 & 24,2823 & & \\
Adaptação & 1 & 204 & 21,4412 & 9425 & 0,001 \\
familiar & 2 & 125 & 19,0400 & & \\
& Total & 329 & & & \\
IPSF total & 1 & 202 & 57,0446 & 10212 & 0,001 \\
& 2 & 124 & 52,2661 & & \\
& Total & 326 & & & \\
\hline
\end{tabular}

O Teste $t$ de Student realizado com autoeficácia e autorregulação indicou diferença estatisticamente significativa com relação a primeira variável. Os participantes do Grupo 1 obtiveram maior média em autoeficácia $(M=23,41$; $D P=3,15)$, quando comparados ao Grupo $2(M=20,71 ; D P=4,84 ; t=2,80, p<$ 01).

A última comparação de grupos que foi realizada objetivou analisar possíveis diferenças entre crianças e adolescentes do sexo masculino (Grupo 1) e feminino (Grupo 2). A Tabela 6 apresenta os resultados encontrados. 
Tabela 6

Resultados da comparação entre os grupos divididos por sexo.

\begin{tabular}{cccccc} 
& $\begin{array}{c}\text { Grupos por } \\
\text { sexo }\end{array}$ & $n$ & Média & $U$ & $p$ \\
\hline Depressão & 1 & 125 & 7,53 & 4901 & 0,001 \\
& 2 & 121 & 11,80 & & \\
Desamparo & Total & 246 & & & \\
& 1 & 73 & 8,04 & 1997,5 & 0,05 \\
& 2 & 71 & 10,47 & & \\
\hline
\end{tabular}

Os grupos foram comparados com relação às variáveis autoeficácia e autorregulação utilizando-se o Teste $t$ Student, e em ambas as variáveis se encontrou diferença estatisticamente significativa. Os participantes do sexo masculino apresentaram maior autoeficácia $(M=22,07 ; D P=4,90 ; t=2,29 ; p<, 05)$ e menor autorregulação $(M=39,72 ; D P=14,26 ; t=4,96 ; p<.001)$ quando comparados às participantes do sexo feminino $(M=20,03$; $D P=4,34$ e $M=49,18$; $D P=17,41$, respectivamente). Em relação aos resultados, recorre-se aos estudos de Baptista et al. (2017), os quais apontaram que diferenças entre os sexos devem buscadas em grupos divididos por idade também, pois diferenças por sexo também estão relacionadas com a idade de forma conjunta. Os autores também apontam que as meninas apresentam mais sintomas internalizantes e os meninos, externalizantes. Para alguns autores (Avanci et al., 2008; Baptista, 2017; Borges et al., 2017), as diferenças entre os sexos podem estar relacionadas à socialização, hormônios e eventos estressantes associados à adolescência (Luby et al., 2014).

\section{CONSIDERAÇÕES FINAIS}

Este estudo representa um primeiro passo exploratório na análise das relações entre indicadores de depressão, autorregulação emocional e percepção do suporte familiar, haja vista por um lado, a escassez de pesquisas semelhantes na literatura nacional e, por outro lado, a necessidade da compreensão da relação entre as variáveis para melhor compreensão do fenômeno da sintomatologia depressiva no público infantojuvenil. Nesse sentido, é importante atentar para os resultados de prevalência de depressão do presente estudo, em que $35 \%$ da amostra total apresentou sintomatologia de moderada a grave.

Mesmo compreendendo que tal variação pode ocorrer, seja por diferenças regionais e pela escolha de instrumentos de medidas, o que chamou atenção foi que uma parte da amostra, composta por crianças e adolescentes de uma cidade do interior paulista, alcançou escores bem mais altos em relação aos demais 
participantes, o que acabou elevando a média final. Tal população está localizada em uma cidade pequena e de poucos recursos financeiros e a escola em que os dados foram coletados, estava situada no subúrbio. Esses dados qualitativos são importantes para presumir que algumas variáveis podem ter interferido nos resultados, as quais não foram controladas, mesmo porque não era objetivo do estudo.

Nesse sentido, uma das limitações do estudo foi não controlar as variáveis sociodemográficas e buscar prevalência para os grupos de diferentes escolas. Além disso, a coleta de dados espiralada não possibilitou algumas análises estatísticas mais específicas, as quais poderiam contribuir de forma mais significativa. Sugere-se novos estudos que controlem esses aspectos mencionados, possibilitando uma análise de possíveis especificidades regionais.

\section{DECLARAÇÃO DE CONFLITO DE INTERESSES}

Não há conflito de interesse.

\section{REFERÊNCIAS}

American Psychiatric Association [APA] (2014). Manual diagnóstico e estatístico de transtornos mentais: DSM-5. $5^{\text {a }}$ ed. Porto Alegre, RS: Artmed.

Argimon, I. I., Terroso, L. B., Barbosa, A. S., \& Lopes, R. M. F. (2013). Intensidade de sintomas depressivos em adolescentes através da escala de depressão de Beck (BDIII) Boletim Academia Paulista de Psicologia, 33(85), 354-372.

Avanci, J. Q., Assis, S. G., \& Oliveira, R. V. (2008). Depressive symptoms during adolescence: A study on psychosocial factors in a sample of teenage students in a city in Rio de Janeiro State, Brazil. Cadernos de Saúde Pública, 24(10), 2334-2346. doi:10.1590/S0102-311X2008001000014

Baptista, M. N. (2009). Inventário de Percepção do Suporte Familiar - IPSF: manual. São Paulo, SP: Vetor.

Baptista, M. N. (2017). Escala Baptista de Depressão - Versão Infanto-Juvenil (EBADEP-IJ). São Paulo, SP: HOGREFE.

Baptista, M. N., Baptista, A. S. D., \& Dias, R. R. (2001). Estrutura e suporte familiar como fatores de risco na depressão de adolescentes. Psicologia Ciência e Profissão, 21(2), 52- 61 . doi:10.1590/S141498932001000200007

Baptista, M. N., Borges, L., \& Serpa, A. L. L. (2017). Gender and age-related differences in depressive symptoms among Brazilian children and adolescents. Paideia, 27(68), 46-53. doi:10.1590/1982-43272768201706 
Baptista, M.N., Carneiro, A.M., \& Sisto, F.F. (2010). Estudo psicométrico de Escalas de Depressão (EDEP e BDI) e o Inventário de Percepção de Suporte Familiar (IPSF). Psicologia em Pesquisa, 4(1), 65-73.

Baptista, M. N., Souza, M. S., \& Alves, G. A. (2008). Evidências de validade entre a Escala de Depressão (EDEP), o BDI e o Inventário de Percepção de Suporte Familiar (IPSF). PsicoUSF, 13(2), 211-220. doi:10.1590/S141382712008000200008

Beck, A. T., Rush, A. J., Shaw, B. F., \& Emery, G. (1997). Terapia Cognitiva da Depressão. Porto Alegre, RS: Artes Médicas.

Borges, L. (2015). Construção da Bateria de Avaliação de Indicadores de Depressão Infantojuvenil - BAID-IJ. (Tese de doutorado). Universidade São Francisco, Itatiba, SP.

Borges, L. \& Baptista, M. N., 2015. Construção e estudos psicométricos da Bateria de Avaliação de Indicadores de Depressão Infantojuvenil (BAID-IJ). (Tese de doutorado). Universidade São Francisco, Itatiba, SP.

Borges, L., Baptista, M. N., \& Serpa, A. L. O. (2017). Structural analysis of depression indicators scale-children and adolescents (BAID-IJ): A bifactorESEM approach. Temas em Psicologia, 25(2), 545-552. doi:10.9788/TP2017. 2-08.

Burwell, R. A., \& Shirk, S.R. (2007). Subtypes of rumination in adolescence: Associations between brooding, reflection, depressive symptoms, and coping. Journal Clinical Child and Adolescent Psychololy, 36(1), 5665. doi:10.1080/15374410709336568.

Coutinho, J., Ribeiro, E., Ferreirinha, R., \& Dias, P (2010). Versão portuguesa da Escala de Dificuldades de Regulação Emocional e sua relação com sintomas psicopatológicos. Revista de Psiquiatria clínica 37(4), 144-151. doi:10.1590/S0101-60832010000400001

Couto, I. S. L., Reis, D. M. L., \& Oliveira, I. R. (2016). Prevalência de sintomas de depressão em estudantes de 11 a 17 anos da rede pública de ensino de Salvador. Revista de Ciências Médicas e Biológicas, 15(3), 370-374. doi:http://dx.doi.org/10.9771/cmbio.v15i3.18205

Cruvinel, M., \& Boruchocitch, E. (2011). Regulação emocional em crianças com e sem sintomas de depressão. Estudos em Psicologia (Natal), 16(3), 219-226.

Dancey, C. P., \& Reid, J. (2013). Estatística sem matemática para psicologia. Porto Alegre, RS: Artmed.

Dessen, M. A. (2010). Estudando a família em desenvolvimento: Desafios conceituais e teóricos. Psicologia: Ciência e Profissão, 30, 202-219.

Ferster, C. B., Culbertson, S., \& Boren, C. P. (1977). Princípios do comportamento. (M. I. R. Silva, M. A. C. Rodrigues, \& M. B. L. Pardo, Trad.). São Paulo, SP: Hucitec.

Girgus, J. S., \& Yang, K. (2015). Gender and depression. Current Opinion in Psychology, 4, 53-60. doi:10.1016/j.copsyc.2015.01.019 
Gonçalves, M., Baptista, M. N., \& Farcas, D. (2016). IPSF: análise da estrutura interna em uma amostra de jovens adultos portugueses. Avaliação Psicológica, 15(1), 115-123. doi:10.15689/ap.2016.1501.12

Gratz, K. L., \& Roemer, L. (2004). Multidimensional assessment of emotion regulation and dysregulation: Development, factor structure, and initial validation of the difficulties in emotion regulation scale. Journal of Psychopathology and Behavioral Assessment, 26(1), 41-54. doi:10.1007/s10862-008-9102-

Howell, A. J., Digdon, N. L., \& Buro, K. (2010). Mindfulness predicts sleep-related self-regulation and well-being. Personality and Individual Differences, 48(4), 419-424. doi: 10.1016/j.paid.2009.11.009

Joormann, J., \& Gotlib, I. H. (2010). Emotion regulation in depression: Relation to cognitive inhibition. Cognition Emotional, 24(2), 281-298. doi.org/10.1080/02699930903407948

Luby, J. L., Gaffrey, M. S., Tillman, R., Abril, L. M., \& Belden, A. C. (2014). Trajectories of preschool disorders to full DSM depression at school age and early adolescence: Continuity of preschool depression. The American Journal of Psychiatric, 171(7), 768-776. doi:10.1176/appi.ajp.2014.13091198

Nelis, D., Quoidbach, J., Hansenne, M., \& Mikolajczak, M. (2011). Measuring individual differences in emotion regulation: The Emotion Regulation ProfileRevised (ERP-R). Psychologica Belgica, 51(1), 49-91. doi:10.5334/pb-51-149

Noronha, A. P. P., \& Baptista, M. N. (2016). Escala de Avaliação da Autorregulação Emocional - EARE. (Relatório técnico não publicado). Universidade São Francisco, Itatiba, SP.

Organização Mundial de Saúde [OMS] (2000). Classificação de Transtornos Mentais e de Comportamento da CID-10: Descrições clínicas e diretrizes diagnósticas. Porto Alegre, RS: Artes Médicas.

Peterson, C., \& Seligman, M. E. P. (2004). Character strengths and virtues: $A$ handbook and classification. New York, NY: Oford University Press/Washington, DC: APA

Porro, M.L., Andres, M.L. \& Rodriguez-Espinola, S. (2012). Regulación emocional y cáncer: utilización diferencial de la expresión y supresión emocional en pacientes oncológicos. Avances en Psicología Latinoamericana, 30(2), 341355.

Rigotto, D. M. (2006). Evidência de validade entre suporte familiar, suporte social e autoconceito. (Dissertação de mestrado). Universidade São Francisco, Itatiba, SP.

Santos, T. M. M. (2006). Evidência de Validade entre Percepção de Suporte Familiar e Traços de Personalidade. (Dissertação de mestrado). Universidade São Francisco, Itatiba, SP. 
Thiengo, D. L., Cavalcante, M. T., \& Lovisi, G. M. (2014). Prevalência de transtornos mentais entre crianças e adolescentes e fatores associados: Uma revisão sistemática. Jornal Brasileiro de Psiquiatria, 63(4), 360-372. doi.org/10.1590/0047-2085000000046

Weinberg, W. A.; Rutman, J.; Sullivan, L.; Penick, E. C., \& Dietz, S. G. (1973). Depression in children referred to an education diagnostic center. Journal of Pediatrics, 83, 1065-1072.

Weiss, N. H., Gratz, K. L., \& Lavender, J. M. (2015). Factor structure and initial validation of a multidimensional measure of difficulties in the regulation of positive emotions: The DERS-Positive. Behavior Modification, 39(3), 431453. doi: $10.1177 / 0145445514566504$

World Health Organization [WHO] (2017). Depression. Recuperado de: http://www.who.int/mediacentre/factsheets/fs369/en/

\section{Sobre as autoras}

Lisandra Borges é psicóloga, Mestre e Doutora pela Universidade São Francisco. É professora no curso de graduação e pós-graduação lato sensu na mesma instituição. Faz parte do Laboratório de Avaliação Psicológica em Saúde Mental LAPSAM-USF. lisandra.borges@usf.edu.br

Janaína Thais Barbosa Pacheco Psicóloga, Mestre e Doutora pelo PPG Psicologia/UFRGS. Realizou Estágio de Pós Doutorado na PUC/RS. É professora do Curso de Psicologia e do Programa de Pós Graduação em Psicologia e Saúde da UFCSPA. Coordenadora do Núcleo de Estudos em Avaliação Psicológica e Intervenções Cognitivas e Comportamentais (NaPsicc) e da Liga Acadêmica de Psicologia Comportamental/UFCSPA. Psicoterapeuta e supervisora clínica. janainapacheco@uol.com.br

A contribuição de cada autor pode ser atribuída como se segue: L.B. e J.T.B.P. participaram de forma equânime na contribuição da escrita do artigo.

Recebido em: 26/10/2017

Revisado em: $12 / 06 / 2018$

Aceito em: 16/06/2018 